\title{
From our homes to yours
}

\author{
The ongoing COVID-19 global pandemic highlights the very human effort that is peer review. We will continue to do \\ all we can to keep the papers flowing and thank our reviewers and authors for their help and understanding under \\ these difficult circumstances.
}

T hese are challenging times. Geoscientists around the world find themselves working from home with labs shut, conferences and fieldwork cancelled, and teaching and learning responsibilities moved online. But this is not just working remotely: this is a pandemic, and each person faces unprecedented disruption to their personal and professional lives. Scientific research is a human effort; it is not immune to such disruption. Peer review, the component of research in which we as a journal play our most active role, is not exempt. However, despite these challenges, it is difficult to argue that geoscience progress can simply be paused until the pandemic passes. For those that are able, we encourage you to continue your research efforts and we will continue to support you as best we can.

Geoscientists are used to preparing for and responding to disasters. We know how threats can be immediate and rapid (in the case of earthquakes, for example), but also drawn-out and seemingly invisible (in the cases of climate change and environmental pollution). Even in a global pandemic, climate change and geohazards - natural and anthropogenic - remain constant threats to the lives of many millions of people around the world. The developing field of geohealth, which seeks to understand how environmental change impacts human health and disease, is directly relevant to the current pandemic: the need for protective equipment for first responders, accurate warning systems, and clear communication of risk are all lessons learned from environmental disasters. There is no shortage of motivation to support continued progress in the geosciences.

However, research depends on many individuals to function efficiently. And these individuals are people too, dealing with unique circumstances brought on by the COVID-19 pandemic. As well as practical challenges of unideal living and working conditions and increased caring responsibilities, many members of the geoscience community are at risk of developing a severe illness themselves, and

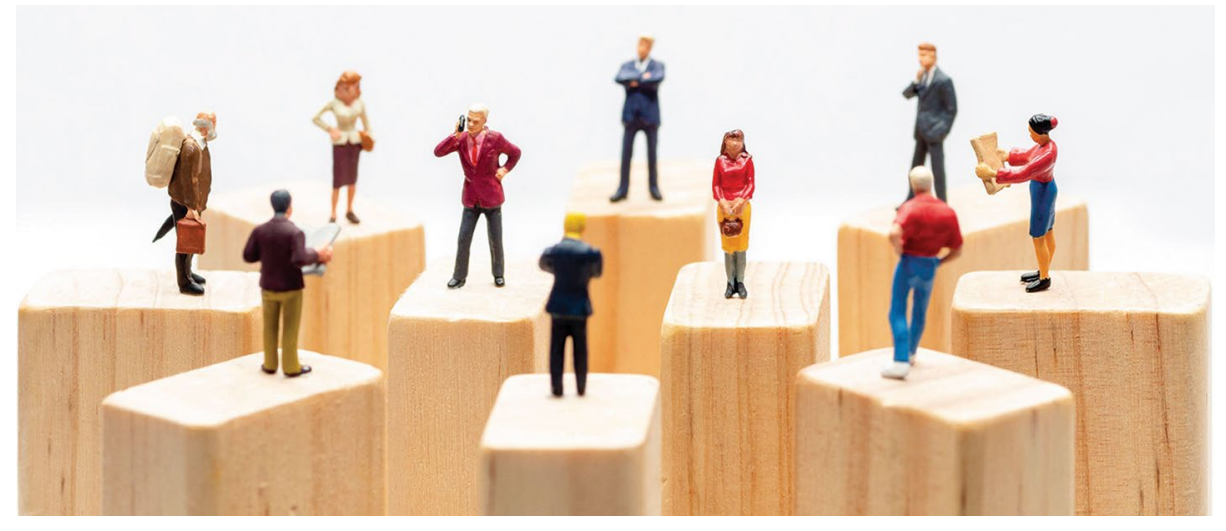

Credit: Ahmad Faizal Yahya / Alamy Stock Photo

we all have close family or friends that are vulnerable. Mental health concerns are also very real in a time of crisis. Interestingly, while being confined at home may seem like the opposite of being in the field, the factors that impact mental health during a lockdown are not dissimilar to those that can be experienced during fieldwork. For example, we may lack privacy, face social and geographical isolation, and experience a heightened sense of danger, all of which can lead to a stressful environment and group dynamic in the field, but also at home.

With the exception of staff at our Shanghai office, which has recently reopened, Nature Geoscience is being run from our homes with all the personal challenges that brings. For the most part, the journal can function relatively smoothly with staff working remotely and we continue to hold the journal to the same high standards. We, the editorial team, are doing our best to keep the journal running as normally as possible. We are also trying to support our authors and reviewers by being more flexible about deadlines, both for authors returning revisions and referees returning their reports.

We understand that not everyone will be able to review right now; even in non-pandemic times, we do not expect everyone to agree to do so all the time. However, if possible, we do kindly ask that you reply to reviewer requests, whether or not you are able to review. Despite the form-like-letter request we send you, editors carefully select and vet potential reviewers; the sooner you let us know you cannot review, the sooner we can find someone else.

We also greatly appreciate suggestions of other possible reviewers, particularly less obvious candidates such as up-and-coming postdocs. Finding enough reviewers for a paper can be challenging, especially at the moment. Many researchers are more available than usual - it is just a question of finding these people.

For those of you who are able to review, we greatly appreciate you lending your time and expertise now, as always. We remind our reviewers about the options available for getting recognition for your work as reviewers for Nature-branded journals.

We are all doing what we can as authors, reviewers and journal staff to help keep the geosciences moving forward in these trying times. But we must also all do what we can to manage our own lives and take care of our loved ones. For some of you, you may be even more productive than before. For others, the science will have to wait, and that is okay. Wherever you are in the world and whatever your personal and professional circumstances, we wish you well and do please take care of yourself.

Published online: 5 May 2020

https://doi.org/10.1038/s41561-020-0580-7 